\title{
Structure and Vibrational Spectra of $\mathrm{Ar}_{\mathrm{n}} \mathrm{H}^{+}(\mathrm{n}=2-3)$
}

\author{
Jake A. Tan and Jer-Lai Kuo
}

Institute of Atomic and Molecular Sciences, Academia Sinica, No.1 Roosevelt Road, Section 4, Da-an District, Taipei City 10617, Taiwan (ROC)

\section{Corresponding Authors}

Jake Tan $\quad$ E-mail: jaketan@gate.sinica.edu.tw

Jer-Lai Kuo E-mail: jlkuo@ pub.iams.sinica.edu.tw 


\section{Table of Contents}

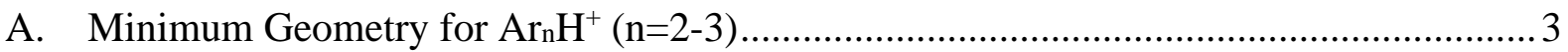

B. $\quad \operatorname{CCSD}(\mathrm{T}) / \mathrm{AVTZ}$ Harmonic Frequencies for $\mathrm{Ar}_{\mathrm{n}} \mathrm{H}^{+}(\mathrm{n}=2-3)$........................................ 4

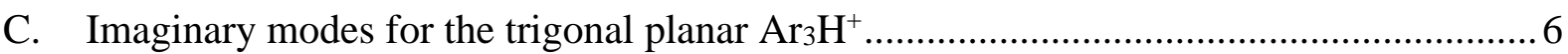

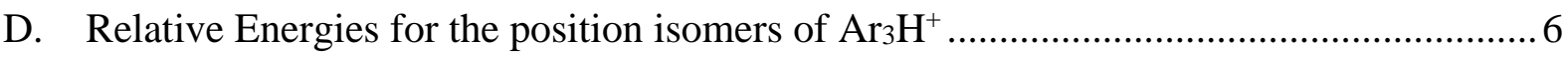

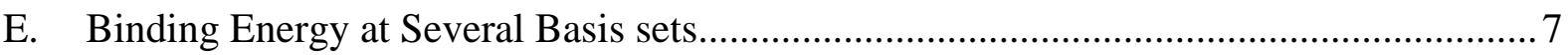

F. Sensitivity of the band intensities for $\mathrm{Ar}_{2} \mathrm{H}^{+}$with respect to the level of theory for the

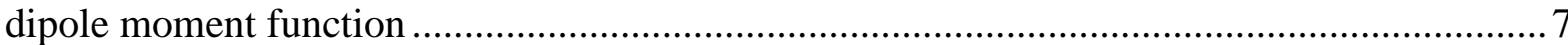




\section{A. Minimum Geometry for $\mathrm{Ar}_{n} \mathrm{H}^{+}(\mathrm{n}=2-3)$}

\section{$\mathrm{Ar}-\mathrm{H}-\mathrm{Ar}$}

Table S1. Minimum Geometry at CCSD(T)/AVTZ

\begin{tabular}{cccc}
\hline $\begin{array}{c}\text { Charge=1, } \\
\text { Mult=1 }\end{array}$ & $\mathbf{X}(\AA)$ & $\mathbf{Y}(\AA)$ & $\mathbf{Z}(\AA)$ \\
\hline $\mathbf{A r 1}$ & 0.000000 & 0.000000 & 1.505819 \\
\hline $\mathbf{A r 2}$ & 0.000000 & 0.000000 & -1.505819 \\
\hline $\mathbf{H}$ & 0.000000 & 0.000000 & 0.000000 \\
\hline
\end{tabular}

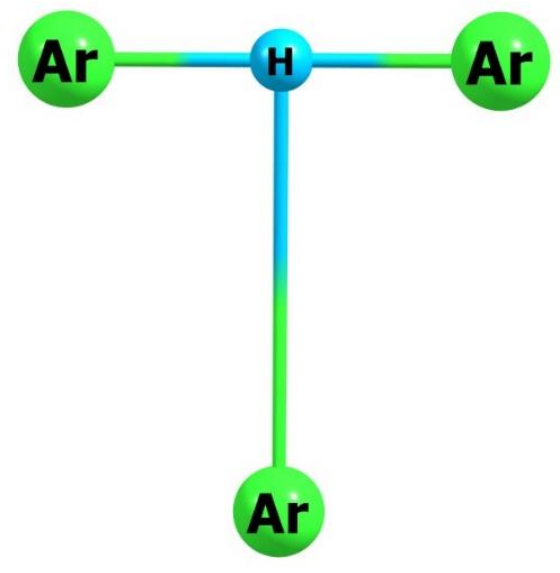

Table S2. Minimum Geometry at CCSD(T)/AVTZ

\begin{tabular}{cccc}
\hline $\begin{array}{c}\text { Charge=1, } \\
\text { Mult=1 }\end{array}$ & $\mathbf{X}(\AA)$ & $\mathbf{Y}(\AA)$ & $\mathbf{Z}(\AA)$ \\
\hline $\mathbf{A r 1}$ & 0.000000 & 1.505691 & -1.056459 \\
\hline $\mathbf{A r 2}$ & 0.000000 & -1.505691 & -1.056459 \\
\hline $\mathbf{A r 3}$ & 0.000000 & 0.000000 & 2.171611 \\
\hline $\mathbf{H}$ & 0.000000 & 0.000000 & -1.056459 \\
\hline
\end{tabular}




\section{$\mathrm{Ar}-\mathrm{H}-\mathrm{Ar}$}

Table S3. Minimum Geometry at CCSD(T)/AVTZ

\begin{tabular}{cccc}
\hline Charge=1, Mult=1 & $\mathbf{X}(\AA)$ & $\mathbf{Y}(\AA)$ & $\mathbf{Z}(\AA)$ \\
\hline Ar1 & 0.000000 & 0.000000 & -3.106371 \\
\hline $\mathbf{A r 2}$ & 0.000000 & 0.000000 & -0.094181 \\
\hline $\mathbf{A r 3}$ & 0.000000 & 0.000000 & 3.287979 \\
\hline $\mathbf{H}$ & 0.000000 & 0.000000 & -1.573695 \\
\hline
\end{tabular}

B. $\operatorname{CCSD}(T) / A V T Z$ Harmonic Frequencies for $\operatorname{Ar}_{n} \mathrm{H}^{+}(\mathrm{n}=2-3)$

Table S4. Harmonic Frequencies for the $\mathrm{Ar}_{2} \mathrm{H}^{+}$.

\begin{tabular}{c|c|c|c|c|}
\hline \multicolumn{3}{c|}{ Ar2 $^{+}$} & \multicolumn{2}{c}{ CCSD(T) } \\
\cline { 3 - 5 } & \multicolumn{2}{c}{ AVTZ } \\
\hline $\begin{array}{c}\text { Mode \# in G09 } \\
\text { Output }\end{array}$ & $\begin{array}{c}\text { Description } \\
\text { Symmetric Ar- } \mathrm{H}^{+} \\
\text {stretch }\end{array}$ & $\begin{array}{c}\text { Symmetry } \\
\text { Label }\end{array}$ & $v\left(\mathrm{~cm}^{-1}\right)$ & $I\left(\frac{\mathrm{km}}{\mathrm{mol}}\right)$ \\
2 & $\Sigma_{g}^{+}$ & 322 & - \\
3 & $\begin{array}{c}\mathrm{H}^{+} \text {bend } \\
\text { Asymmetric Ar- } \mathrm{H}^{+} \\
\text {stretch }\end{array}$ & $\Pi_{u}$ & 706 & - \\
\hline
\end{tabular}


Table S5. Harmonic Frequencies for the T-shaped $\mathrm{Ar}_{3} \mathrm{H}^{+}$.

\begin{tabular}{|c|c|c|c|c|}
\hline \multirow{2}{*}{\multicolumn{3}{|c|}{$\mathrm{Ar}_{3} \mathrm{H}^{+}(\mathrm{T}$-shaped $)$}} & \multirow{2}{*}{\multicolumn{2}{|c|}{$\frac{\operatorname{CCSD}(\mathrm{T})}{\mathrm{AVTZ}}$}} \\
\hline & & & & \\
\hline $\begin{array}{c}\text { Mode \# in G09 } \\
\text { Output }\end{array}$ & Description & $\begin{array}{l}\text { Symmetry } \\
\text { Label }\end{array}$ & $v\left(\mathrm{~cm}^{-1}\right)$ & $I\left(\frac{\mathrm{km}}{\mathrm{mol}}\right)$ \\
\hline 1 & Frustrated rotation & $B_{2}$ & 44 & - \\
\hline 2 & $\begin{array}{c}\text { Intermolecular } \\
\operatorname{Ar} . \mathrm{Ar}_{2} \mathrm{H}^{+} \text {stretch }\end{array}$ & $A_{1}$ & 57 & - \\
\hline 3 & $\begin{array}{l}\text { Symmetric core-ion } \\
\text { Ar- } \mathrm{H}^{+} \text {stretch }\end{array}$ & $A_{1}$ & 321 & - \\
\hline 4 & In-plane $\mathrm{H}^{+}$bend & $A_{1}$ & 686 & - \\
\hline 5 & Out-of-plane $\mathrm{H}^{+}$bend & $B_{1}$ & 706 & - \\
\hline 6 & $\begin{array}{l}\text { Asymmetric core-ion } \\
\text { Ar- } \mathrm{H}^{+} \text {stretch }\end{array}$ & $B_{2}$ & 946 & - \\
\hline
\end{tabular}

Table S6. Harmonic Frequencies for the linear $\mathrm{Ar}_{3} \mathrm{H}^{+}$.

\begin{tabular}{|c|c|c|c|c|}
\hline \multirow{2}{*}{\multicolumn{3}{|c|}{$\mathbf{A r}_{3} \mathbf{H}^{+}($Linear $)$}} & \multirow{2}{*}{\multicolumn{2}{|c|}{$\frac{\operatorname{CCSD}(\mathrm{T})}{\mathrm{AVTZ}}$}} \\
\hline & & & & \\
\hline $\begin{array}{l}\text { Mode \# in G09 } \\
\text { Output }\end{array}$ & Description & $\begin{array}{c}\text { Symmetry } \\
\text { Label }\end{array}$ & $v\left(\mathrm{~cm}^{-1}\right)$ & $I\left(\frac{\mathrm{km}}{\mathrm{mol}}\right)$ \\
\hline 1 & Frustrated Rotation & $\Pi$ & 13 & - \\
\hline 2 & $\begin{array}{c}\text { Intermolecular } \\
\text { Ar...A. }{ }_{2} \mathrm{H}^{+} \text {stretch }\end{array}$ & $\Sigma^{+}$ & 52 & - \\
\hline 3 & $\begin{array}{l}\text { Symmetric core-ion } \\
\text { Ar- } \mathrm{H}^{+} \text {stretch }\end{array}$ & $\Sigma^{+}$ & 315 & - \\
\hline 4 & $\mathrm{H}^{+}$bend & $\Pi$ & 708 & - \\
\hline 5 & $\begin{array}{l}\text { Asymmetric core-ion } \\
\text { Ar- } \mathrm{H}^{+} \text {stretch }\end{array}$ & $\Sigma^{+}$ & 981 & - \\
\hline
\end{tabular}




\section{Imaginary modes for the trigonal planar $\mathrm{Ar}_{3} \mathrm{H}^{+}$}

a)

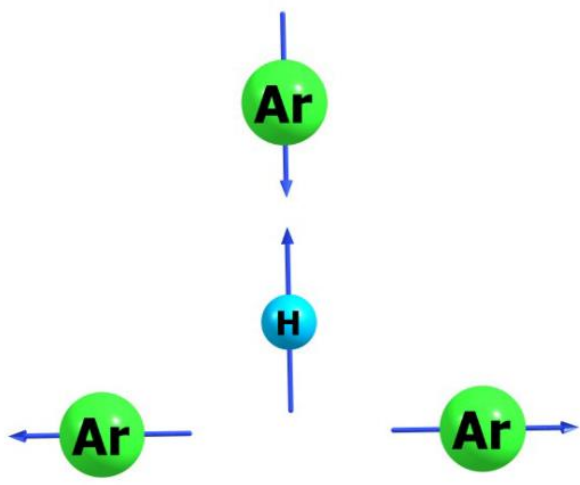

b)

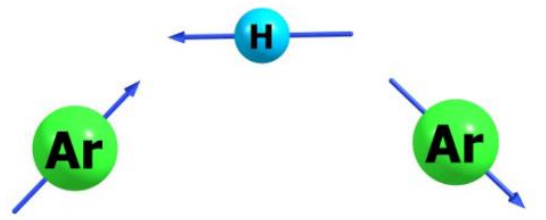

Figure S1. Imaginary modes for the trigonal planar $\mathrm{Ar}_{3} \mathrm{H}^{+}$. These modes are degenerate and has an imaginary frequency of $1025 i \mathrm{~cm}^{-1}$ at the $\operatorname{CCSD}(\mathrm{T}) / \mathrm{AVTZ}$ level of theory and basis.

\section{Relative Energies for the position isomers of $\mathrm{Ar}_{3} \mathrm{H}^{+}$}

Table S7. CCSD(T)/AVTZ relative energies at $0 \mathrm{~K}$ in $\mathrm{kcal} / \mathrm{mol}$ between the $\mathrm{T}$-shaped and linear isomers of $\mathrm{Ar}_{3} \mathrm{H}^{+}$.

\begin{tabular}{c|c|c|c|c}
\hline \multirow{2}{*}{ CCSD(T) } & \multicolumn{2}{|c|}{$\begin{array}{c}\text { Without ZPE } \\
\text { correction }\end{array}$} & \multicolumn{2}{c}{ With ZPE correction } \\
\cline { 2 - 5 } & T-shaped & Linear & T-shaped & Linear \\
\hline \multirow{2}{*}{ AVTZ } & 0.000 & 0.673 & 0.000 & 0.717 \\
\hline
\end{tabular}

Note: ZPE correction is based on harmonic analysis. 


\section{E. Binding Energy at Several Basis sets}

Using the optimized geometries at the CCSD(T)/AVTZ level of theory and basis, single point calculations at AVQZ and AV5Z basis sets were performed. For each species the energies for these three basis sets were used to do the complete basis set extrapolation.

Table S8. Binding Energy calculated at the CCSD(T) level of theory with several basis sets.

\begin{tabular}{c|cccc}
\hline \multirow{2}{*}{ Process } & \multicolumn{4}{|c}{ CCSD(T) Binding Energy (kcal/mol) } \\
\cline { 2 - 4 } & AVTZ & AVQZ & AV5Z & CBS \\
\hline$A r H_{(g)}^{+} \rightarrow A r_{(g)}+H_{(g)}^{+}$ & 94.783 & 93.964 & 93.977 & 93.643 \\
$A r_{(g)}+A r H_{(g)}^{+} \rightarrow A r_{2} H_{(g)}^{+}$ & 15.613 & 15.556 & 15.462 & 15.417 \\
$A r_{(g)}+A r H_{(g)}^{+} \rightarrow A r_{2} H_{(g)}^{+}(T-$ shaped $)$ & 1.988 & 2.001 & 2.040 & 2.049 \\
$A r_{(g)}+A r H_{(g)}^{+} \rightarrow A r_{2} H_{(g)}^{+}($Linear $)$ & 1.316 & 1.318 & 1.343 & 1.344 \\
\hline
\end{tabular}

\section{F. Sensitivity of the band intensities for $\mathrm{Ar}_{2} \mathrm{H}^{+}$with respect to the level of theory for the dipole moment function}

To explore how sensitive are the calculated intensities with respect to the level of theory for the dipole moment function, we have performed anharmonic calculations using the same potential energy surface but different levels of theories for the dipole moment functions. We found that the results for these systems are less sensitive with the level for DMF. 


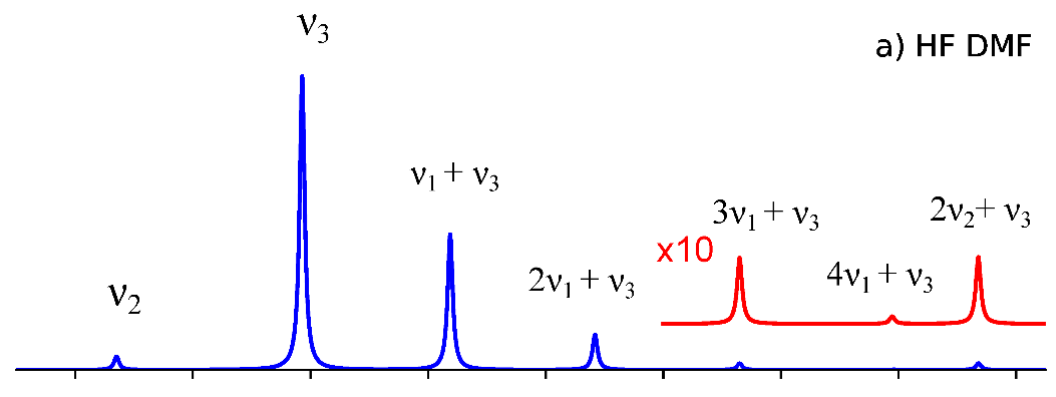

b) MP2 DMF

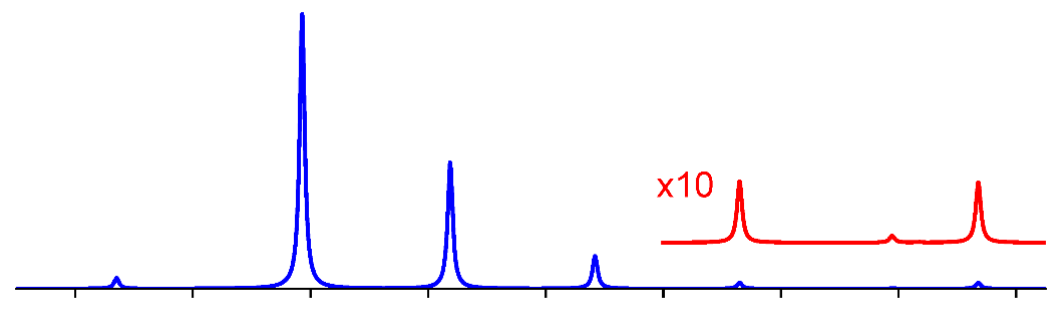

c) CCSD DMF

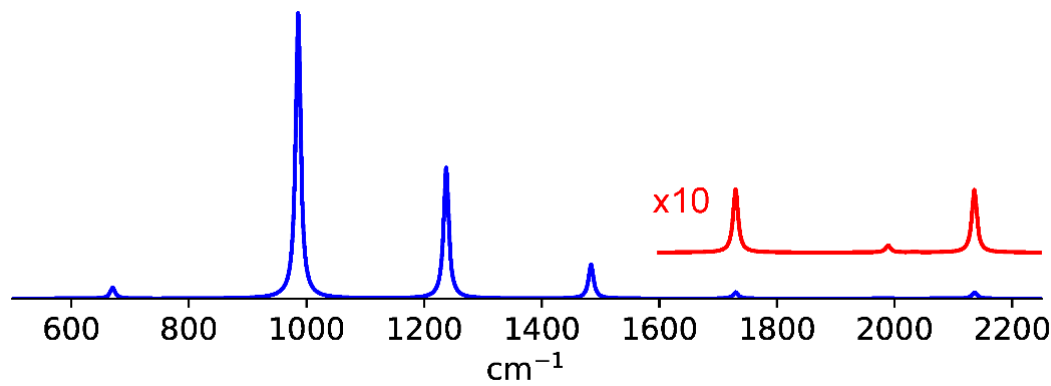

Figure S2. Anharmonic spectrum for $\mathrm{Ar}_{2} \mathrm{H}^{+}$calculated using a CCSD(T)/AVTZ PES and several levels of dipole moment functions at the same basis set. a) HF, b) MP2, and c) CCSD

Table S9. Peak positions and their corresponding intensities for the anharmonic calculation using CCSD(T)/AVTZ PES and several DMF.

\begin{tabular}{|c|c|c|c|c|}
\hline \multirow[t]{2}{*}{ Assignment } & \multirow{2}{*}{$\begin{array}{c}\text { Peak Position } \\
\left(\mathrm{cm}^{-1}\right)\end{array}$} & \multicolumn{3}{|c|}{ Intensity $(\mathrm{km} / \mathrm{mol})$ using Dipole Moment Function } \\
\hline & & $\mathbf{H F}$ & MP2 & CCSD \\
\hline$v_{1}$ & 292 & 0 & 0 & 0 \\
\hline$v_{2}$ & 670 & 67 & 53 & 54 \\
\hline$v_{3}$ & 986 & 2904 & 2706 & 2822 \\
\hline$v_{1}+v_{3}$ & 1237 & 1339 & 1241 & 1294 \\
\hline $2 v_{1}+v_{3}$ & 1484 & 349 & 323 & 336 \\
\hline $3 v_{1}+v_{3}$ & 1730 & 65 & 60 & 62 \\
\hline $4 v_{1}+v_{3}$ & 1989 & 7 & 7 & 7 \\
\hline $2 v_{2}+v_{3}$ & 2136 & 66 & 59 & 62 \\
\hline
\end{tabular}

